\title{
Reducing Symptoms in Chronic Pulmonary Patients with Massage, Acupuncture, and Guided Imagery
}

\author{
Tracy Fasolino PhD, FNP-BC, ACHPN*, Mary Margaret Cain, Heather Hufnagel and Haley McKee \\ Associate Professor, Clemson University, USA
}

*Corresponding author: Tracy Fasolino, Associate Professor, Clemson University, USA.

Received Date: April 22, 2019

Published Date: June 06, 2019

Abbreviations : COPD: Chronic Obstructive Pulmonary Disease; QOL: Quality of Life; CPD: Chronic Pulmonary Disease; CAM: Complementary and Alternative Medicines; MT: Massage Therapy; GI: Guided Imagery; AQLQ: Asthma Quality of Life Questionnaire; SAS: Self-Rating Anxiety Scale; PNS: Parasympathetic Nervous System

\section{Introduction}

Chronic pulmonary diseases, such as chronic obstructive pulmonary disease (COPD), chronic bronchitis, pulmonary fibrosis, and asthma, affect 40 million people in the United States [1]. As these diseases progress, patients experience uncontrolled symptoms, such as dyspnea (shortness of breath), anxiety, and pain. Furthermore, patients and the caregivers describe decreased quality of life (QOL). Management of chronic pulmonary disease (CPD) focus on pharmaceutical interventions with medicines to open and relax the smooth muscles of the airway, thin secretions, and decrease inflammation. At times, these medications may worsen patient symptoms or cause unwanted side effects, such as tremors associated with short-acting beta agonists [2] or weakened respiratory muscles with corticosteroid use [3]. Other therapies, such as pulmonary hygiene and postural drainage are often uncomfortable and worsen dyspnea. Certain pulmonary rehabilitation exercises targeting the shoulder muscles can also make breathing more difficult [4].

When prescribed medications and therapies are no longer effective, the patient, caregiver, and healthcare team struggle to find ways to help reduce the burden of the disease. Physicians, Nurse Practitioners, and Physician Assistants may opt to introduce opiates and anti-anxiety medications for patients with uncontrolled dyspnea. However, patients and family members are familiar with the stigma and skepticism related to these therapies. Society associates addiction, dependence, and adverse drug effects with opiates and anti-anxiety medications. The challenge is finding other therapies that can reduce dyspnea without introducing unwanted side effects. Integrating massage therapy, acupuncture, and/or guided imagery into routine care for patients with CPD could reduce symptoms and likely decrease use of opiates and antianxiety medications. Massage therapy, acupuncture, and guided imagery are relatively easy to integrate, have few associated side effects, and can be used as an adjunct for controlling physiological and psychological impact as part of complementary and alternative medicines (CAM) [5]. Furthermore, caregivers can become an active part of the management by learning how to administer these therapies.The objective of this paper is to present findings of the systematic review of the role of massage therapy, acupuncture, and guided imagery for patients with CPD. The basic methods of each therapy, impact on CPD related symptoms, and associated precautions will be covered.

\section{Methods}

The review of the literature was protocol-based and conducted in accordance with the PICO [6] and the PRISMA recommendations for reporting systematic reviews [7]. We conducted the review using a keyword search in the electronic databases of PubMed, Psych INFO, Web of Science, Cochrane Library and CINAHL. All databases were searched using relevant MeSH terms. Keywords related to the population (Chronic pulmonary disease OR chronic obstructive pulmonary disease OR COPD OR asthma OR chronic bronchitis OR emphysema) were combined with key words related to the intervention (massage therapy OR acupuncture OR guided imagery OR mindfulness OR relaxation OR meditation) and outcomes (dyspnea OR shortness of breath OR breathlessness OR 
fatigue $\mathrm{OR}$ anxiety OR panic OR quality of life OR functional status). The search was conducted by each author on the specific topic with the primary author reviewing all results. Initially, articles were to be extracted with the past 5 years (2014-2019). Due to a low number of relevant articles, we expanded the search to the past 15 years.

\section{Selection Procedure and Data Extraction}

Only English-language reports published in peer-reviewed journals were considered eligible for the present study. Eligible studies were those that evaluated individual, or group-based MT, acupuncture, and GI interventions aimed at alleviating symptoms associated with CPD, including the physiological and psychological impact. Papers were excluded if the concentration was on the use of other CAM therapies, such as hypnosis. Initially, we focused on designs that utilized control groups. The search results did not yield adequate articles, so we expanded to include all designs.

In the first round of assessment, the primary author (TF) discussed the articles extracted with the other authors (MC, HH, HM). The team identified and removed duplicates and screened the titles and abstracts of the identified references with the purpose of excluding irrelevant studies. In the second round of assessment, full texts of the remaining references were read, and ineligible reports were excluded. Disagreements and uncertainties were discussed until a negotiated conclusion was reached. Using a Microsoft Excel spreadsheet, essential elements were extracted and organized. Data from the included studies were extracted independently and cross-checked by the primary author. The initial search yielded 184 articles, out of which 48 articles were screened to match with inclusion criteria. The authors agreed on 20 articles for the systematic review, keeping in mind the broad and complex nature of the field of MT, acupuncture, and GI interventions.

\section{Massage Therapy}

Massage therapy (MT) is the art of manipulation of the body in a systematic manner using light to moderate pressure and focusing on key regions [8]. While studies have shown patients enjoy MT, the 2012 National Health Interview Survey (NHIS) found only $9.7 \%$ of patients receive the therapy [9]. The healthcare community supports MT with more than $70 \%$ of providers referring patients to massage therapists as an adjunct to care management [10]. Massage therapy improves blood flow, oxygenation, and heart rate; and decreases fluid and toxic waste buildup in the lymphatic system [11,12]. Furthermore, MT has demonstrated improvement in psychological well-being [13].

There are various types of massage, such as neuromuscular release massage therapy (NMRT), hot stone massage, Swedish massage, and others. Neuromuscular release massage therapy is a specialized form of deep tissue massage using pressure and friction on specific regions to eliminate symptoms. This type of massage is ideal for patients with CPD, particularly in regions of the upper back. Hot stone massage incorporates smooth, flat stones composed of a type of volcanic rock that holds heat. These stones are placed in various regions of the body and can be actively used during the massage. A Swedish massage involves rubbing body muscles with long gliding strokes in the direction of blood returning to the heart. As defined by Beeken et al. [14], MT methods require the application of pressure and resistance to muscle groups that are hypertonic, injured, or spasming to promote healing.

Studies using MT for CPD patients considered three major aspects:

1. Massage at a set time daily or weekly.

2. The type of massage.

3. Provide measurable values (i.e. peak flow values, pulmonary function tests, and FVC values) $[14,15]$.

The use of MT over an extended period proves to be beneficial for patients experiencing dyspnea. Improvements in peak flow values and oxygen saturation (SPO2 increased by 1.62\%) have been recorded [14]. Pulmonary function tests (FVC and FEV1 values) and the Borg Visual Analog Scale (rating scale of 0-10 with 10 being maximal discomfort) improved with the use of MT. Furthermore, participants had increases in energy and decrease in dyspnea as measured by the Borg Visual Analog Scale [14].

Most published studies examining the value of MT involve pediatric patients with asthma. Asthma affects $8.3 \%$ of the US pediatric population (Center for Disease Control, 2018), and often parents are open to the idea of CAM therapies to help their child. In fact, approximately $71 \%$ of parents said they would consider complementary care for their asthmatic child [16]. Studies have found physiological and psychological improvements with use of MT in pediatric asthma patients [17-19]. Nekooee et al., (2008) studied the benefits of a 20-minute daily bedtime massage over a period of one month. They found an overall improvement in pulmonary spirometry, airway tonicity and sensitivity as well as reduced anxiety levels in pediatric patients.

In the few published studies for adult patients, MT helped reduce dyspnea and improve QOL. Beeken et al. (1998) studied the impact of MT over a 6-month period in patients between the age of 5774. The patients had less dyspnea, improvements in physiological measurements (peak flows, pulmonary function tests, and thoracic gas volume) and self-reported QOL. Patients receiving MT reported high satisfaction with their treatment as shown by $40 \%$ of patients voluntarily continuing treatment at their own expense after the trial had ended [14]. Exercise intolerance is common in patients diagnosed with moderate to severed CPD. In fact, approximately $50 \%$ of CPD patients report exercise intolerance [20]. Using MT can improve bronchodilation in CPD patients allowing greater oxygenation with effects similar to pharmaceutical bronchodilators [21].

In addition to reducing dyspnea, MT has shown benefits in reducing pain, anxiety and depression which are common complaints reported by patients with CPD linked to neurotransmitter levels. A few studies have measured neurotransmitter (serotonin, dopamine, and norepinephrine) and corticosteroid levels (cortisol) [22,23]. Patients receiving MT have increased levels of serotonin and 
decreased levels of dopamine as compared to patients not receiving MT, resulting in alleviation of pain and improvement in mood. Furthermore, lower levels of cortisol have been noted after MT which result in less stress and anxiety [24].

The safety of MT has received little attention, with only case reports describing adverse effects of the intervention. The type of massage utilized was more aggressive and involved painful procedures. In traditional MT, such as the Swedish massage, side effects include soreness, bruising, and discomfort. However, these issues occur in less than $1 \%$ of the patients [25]. There are very few side effects associated with MT. The positive effects of MT, such as improved exercise tolerance and peak flow improvement, began immediately and lasts greater than 48 hours [25].

Massage therapy has the potential to be used universally in pediatric and adult populations with variation in styles and frequencies. Current literature supports the role of MT to reduce the symptom burden associated with CPDs. The potential biochemical changes and pulmonary function improvement show that MT has beneficial effects for the participants with few unwanted side effects.

\section{Acupuncture}

Acupuncture is an ancient Chinese needling technique used by practitioners for centuries to correct an imbalance or deficiency in "chi," or life force energy. When homeostasis is altered, imbalances can be manifested through the physical, spiritual, or emotional systems. Different types of acupuncture exist, including traditional acupuncture, non-needle acupuncture, acupressure, and many others. The basic premise of acupuncture interventions is to stimulate the body's neurohormonal pathways to release chemicals known as neurohormones [26]. Neurohormones are substances secreted by specialized cells within the nervous system, rather than the endocrine system. Two examples of neurohormones, enkephalins and endorphins, possess similar properties to morphine and other analgesics [27].

Traditional acupuncture requires placement of fine, stainless steel needles into specific areas on the body to elicit neurohormone release. Licensed acupuncturists maneuver needles to elicit a "de qui" response and restore balance in homeostasis. Non-needle acupuncture involves placement of electrodes on acupuncture points to stimulate specific nerves, which causes neurohormone release. An example of a non-needle acupuncture is a transcutaneous electrical nerve stimulation (TENS) unit. This system uses electrodes over acupuncture points to deliver low frequency electrical impulses that block or suppress messages back to the brain. The non-needle acupuncture is particularly appealing because patients only feel a tingling or slight burning sensation on their skin [28].

Studies investigating acupuncture have reported improvements in dyspnea for patients with CPD. Brinkhaus et al. [29] studied the benefits of 15 acupuncture sessions over a 3-month period in patients with chronic asthma. The study assessed the patient's symptoms, level of activities, and stabilization of emotions using the Asthma Quality of Life Questionnaire (AQLQ). Patients receiving acupuncture reported improvements in symptoms and had higher scores on AQLQ compared to those patients not receiving the therapy. Other studies report improvements in pulmonary function values and decreased symptoms when using acupuncture [30]. Similar to MT, the benefits of acupuncture appear with repeated treatments instead of one treatment. Marino et al. (2010) conducted a one-session acupuncture intervention on six individuals diagnosed with CPD. While the intervention did not reduce dyspnea, patients reported reduced work of breathing and demonstrated improvements in the amount of air exhaled.

Acupuncture has demonstrated benefits in reducing the heart rate (HR). For example, Ngai et al. (2013) and Marino et al. (2010) studies reported significant therapeutic reductions in HR following acupuncture therapy. In a case study, Ngai et al. (2013) [31] found the HR of a 74-year-old Chinese male decreased from 98 beats per minutes (bpm) to 86bpm after Acu-TENS therapy. The patient sustained the lower HR for 45 minutes post-intervention. While not all reviewed studies considered effects on HR, the psychological symptom of anxiety has been associated with elevations in HR. In a study by $\mathrm{Hu}, \mathrm{Wu}$, and Hou (2017), acupuncture was correlated with reduction in self-reported anxiety. The researchers measured anxiety using Self-Rating Anxiety Scale (SAS) in patients with lung cancer experiencing dyspnea. Self-reported QOL has also been considered by several studies, using scales such as the Short-Form 36, the Asthma Quality of Life Questionnaire (AQLQ) and the QOL scale for adult Korean asthmatics [29] reported improvements in mean scores of the AQLQ in patients having received acupuncture.

There are reports of side effects and discomfort with acupuncture, though none are life threatening. In a study of 1,145 patients, approximately $11.5 \%$ of the patients reported side effects of pain with needle insertion, minor local bleeding, sweating, dizziness and decrease in blood pressure [29]. The risk of bleeding increases with patients on anticoagulant therapy or those with known bleeding disorders. While the incidence of infection from needle insertion with acupuncture is extremely low, there is a risk if sterilization techniques are faulty.

\section{Guided Imagery}

Guided imagery (GI) is a form of meditation and involves all the senses, not just the visual aspect. Other terms for GI are visualization, mental rehearsal and mental imagery [32]. As a relaxation exercise, GI connects physiological with psychological aspects using behavioral-based interventions to reduce symptoms, such as anxiety, pain, and stress $[33,34]$. In additional to psychological improvement, GI helps to reduce blood pressure, heart rate, respiratory rate, and body temperature [35]. The purpose of GI is to connect the mind, body, and spirit; the goal is to heal through this connectivity using all the senses (Academy of Guided Imagery, 2019). Effective GI requires the ability to incorporate one's own imagination to influence positive impact on well-being. Guided imagery can be incorporated into the clinical setting for specific 
reasons, such as: 1) creating individualized GI intervention based upon specific needs; 2) preparing a patient for an upcoming diagnostic procedure; and 3) helping produce a physiologic effect, such as relaxation or anxiety reduction.

Theories underlying the physiological impact of GI consider the neurological \& chemical systems of the body. Investigators propose that GI activates the parasympathetic nervous system (PNS), thereby reducing the blood pressure and heart rate (Hyland et al., 2016). There is also evidence that activating the PNS improves the respiratory system [36]. For example, when skeletal muscles relax, there is a decrease demand on the respiratory system. A state of relaxation as a result of GI affects the levels of endorphins (natural analgesic) and reduces pain experience. Higher levels of endorphins reduce perceptions of pain and trigger positive feeling in the body [36]. Additionally, AuBuchon [37] proposes that GI provides dyspnea relief by serving as a cognitive distraction for the patients.Research suggests that negative stimuli, when presented, initiate cognitive negative responses to the feelings experienced from dyspnea. The use of GI targets the sense of helplessness and hopelessness that is characteristic of CPD patients may be able to effectively reduces these negative emotions.

Studies examining the benefits of GI for dyspnea in chronic pulmonary conditions are sparse, but supportive. Current research on the benefits of GI are focusing on heart failure, a similar chronic disease with debilitating symptoms and impact on QOL. Louie [38] found benefits of GI in COPD patients by measuring the oxygen saturation levels (SPO2). Commonly, oxygenation levels are lower in patients with CPDs. During the study, participants received training on the technique of GI. After six individual, 30-minute sessions of GI, the physiological measurements of SPO2, HR, upper thoracic surface electromyography, skin conductance and peripheral skin temperature were recorded. Participants involved in the GI intervention demonstrated statistically significant improvements in oxygen saturations.

Two pilot studies investigated GI benefits in patients with chronic asthma, considering the emotional and physical elements. Freeman and Welton [39] compared GI and critical thinking techniques in adult asthma patients on symptoms (wheezing, coughing, etc.) and self-report measures, such as Profiles of Mood States. Participants engaged in either GI or critical thinking techniques for two 2-hour sessions over a period of 6 weeks. Guided imagery demonstrated greater benefits for physiological and psychological measurements in patients with asthma. Similarly, Epstein [40] examined the benefits of GI in adult asthma patients. Participants utilized 7 different GI exercises three times a day for a total of 15 minutes. The outcome measures included pulmonary spirometry, frequency of medication use, depression, anxiety, and QOL. The study results did not find significant improvements in the outcome measures in participants using GI techniques.

The benefits of GI include low cost of treatment and the portability of the therapy. Patients and caregivers can administer this therapy with minimal supervision. However, integrating GI does require complex cognition. This applies to both the patient and the caregiver performing the therapy. Guided imagery is most successful in regulating breathing by relaxation when patient's are under stress or panic. Some studies have found that GI can lead to false memories; any incident or an event that did not actually occur. These delusions are supported by the visual virtual simulations incorporated within GI techniques [41]. A minimally invasive procedure, GI practice is generally considered safe for most people [42]. Improving the patient's sense of empowerment through GI techniques to reduce symptoms promotes changes in perceptions of illness and overall well-being.

\section{Discussion}

Complementary \& alternative medicine (CAM) is rapidly expanding within the US. The World Health Organization (2013) defines CAM therapy as "a broad set of health practices that are not part of that country's own tradition or conventional medicine and are not fully integrated into the dominant health care system." The scope of these therapies extends from natural products to mind and body practices. As nurses, we should offer CAM therapies alongside of conventional medicine for a patient with CPD in order to support and dispel misconceptions of the use of these interventions. Our review selected three specific treatments (MT, acupuncture, and GI) due to the potential of integration into the care plan for patients with CPD, particularly in end-stage disease. Each of these therapies connect the physiological and psychosocial aspects of dealing with chronic illnesses to alleviate and reduce the symptom burden.

Current small-scale pilot studies support various aspects of MT, acupuncture, and GI for symptom management during- and posthospitalization. The challenge may be identifying practitioners that can offer these services to patients. Interestingly, prior to expansion of scientific studies care and pharmaceutical discoveries, nurses provided MT as a basic comfort measure for patients. Archived nursing textbooks describe the procedure, steps, and outline the value of MT. Nurses should refocus attention on the healing of the mind \& body and reinforce the value of touch into practice.

Integrating acupuncture into the acute care setting poses greater challenges given the specialized training required. Nurses can certainly advocate for the use of acupuncture or TENS units as an intervention, particularly when conventional therapies are unsuccessful. Guided imagery is the easiest CAM therapy to incorporate compared to MT and acupuncture but requires complex cognition and concentration by the patient. The side effect profiles of MT, acupuncture, and GI are minimal. Each of these interventions need consistent, long-term use to reduce dyspnea in patients with CPD. Massage therapy, acupuncture, and GI can be part of the standard care and scheduled, just like the medication administration.

These alternative, nonpharmacologic treatments show promise on a small scale. When attempting to reduce opiate and anti-anxiety medication use, MT, acupuncture, and GI have not been evaluated and comparative studies are lacking. As the opiate epidemic escalates, alternative therapies are needed. The CAM 
therapies discussed can be integrated by the patient's caregivers and allows them to become active participants in care. The costs of incorporating the therapies are relatively low; the adverse effects are minimal; and outcomes of integration are positive. As advocates and trusted caregivers, nurses are uniquely positioned to bring MT, acupuncture, and GI into care planning for patients with CPD.

\section{Conflict of Interest}

No conflict of interest.

\section{Acknowledgement}

None.

\section{References}

1. American Lung Association (2018). Estimated prevalence and incidence of lung disease.

2. Cazzola M, Matera MG (2012) Tremor and $\beta$ (2)-adrenergic agents: is it a real clinical problem? Pulmonary Pharmacology \& Therapeutics 25(1): $4-10$

3. Foye PM, Lim S (2018) Corticosteroid-Induced Myopathy.

4. Holland AE, Wadell K, Spruit MA (2013) How to adapt the pulmonary rehabilitation programme to patients with chronic respiratory disease other than COPD. European Respiratory Review 22(130): 577-86.

5. Lee AL, Harrison SL, Goldstein RS, Brooks D (2018) An exploration of pain experiences and their meaning in people with chronic obstructive pulmonary disease. Physiother Theory Pract 34 (10): 765-772.

6. Sackett DL, Richardson WS, Rosenberg W, Haynes RB (1997) EvidenceBased Medicine: How to Practice and Teach EBM. Churchill Livingston, New York, USA

7. Moher D, Liberati A, Tetzlaff J, Altman DG (2009) Preferred reporting items for systematic reviews and meta-analyses: the PRISMA statement. J Clin Epidemiol 62: 1006-1012.

8. Kennedy AB, Cambron JA, Sharpe PA, Travillian RS, Saunders RP (2016) Clarifying definitions of the massage therapy profession: The results of the Best Practices Symposium, Int J Ther Massage Bodywork 9(3): 1526.

9. Falci L, Shi Z, Greenlee H (2016) Multiple chronic conditions and use of complementary and alternative medicine among US adults: Results from the 2012 National Health Interview Survey. Preventing Chronic Disease 12(61): 1-13.

10. Verhoef MJ, Page SA (1998) Physicians perspectives on massage therapy. Can Fam Physician 44: 1018-1020.

11. Clarke S, Munro P, Annemarie L (2019) The role of manual therapy in patients with COPD. Healthcare 7(1): 21-32.

12. Ferrell Torry AT, Glick OJ (1993) The use of therapeutic massage as a nursing intervention to modify anxiety and the perception of cancer pain. Cancer Nursing 16(2): 93-101.

13. Field T (2016) Massage therapy research review. Complementary Therapies in Clinical Practice 24: 19-31.

14. Beeken JE, Parks D, Cory J, Montopoli G (1998) The effectiveness of neuromuscular release massage therapy in five individuals with chronic obstructive lung disease. Clinical Nursing Research 7(3): 309-325.

15. Neekoee A, Faghihinia J, Ghasemy R, Ghaibizadeh, M Ghazavi Z (2008) Effect of massage therapy on children with asthma. Iran Journal of Pediatrics 18(2): 99-130.

16. Ernest E (1998) Use of complementary therapies in childhood asthma. Pediatric Asthma, Allergy \& Immunology 12(1): 29-32.

17. Fattah MA, Hamdy B (2011) Pulmonary functions of children with asthma improve following massage therapy. The Journal of Alternative \& Complementary Medicine 17(11): 1065-1068.
18. Zhu S, Wang N, Wang D (1998) Clinical investigation on massage for prevention and treatment of recurrent respiratory tract infection in children. Journal of Traditional Chinese Medicine 18: 285-291.

19. Neekoee A, Faghihinia J, Ghasemy R, Ghaibizadeh, M Ghazavi Z (2008) Effect of massage therapy on children with asthma. Iran Journal of Pediatrics 18(2): 99-130.

20. Saey D, Debigaré R, Le Blanc P, Mador MJ, Côté H, et al. (2003) Contractile leg fatigue after cycle exercise: A factor limiting exercise in patients with COPD. Am J Respir Crit Care Med 168(4): 425-430.

21. Turchaninov R (2009) How massage therapy heals the body: Part III. Science of Massage Institute.

22. Bicikova M, Macova L, Kolatorova L, Hill M, Novotny J, et al. (2018) Physiological changes after spatreatment-a focus on endocrinology. Physiological Research 28(67Supplement 3): S525-S530.

23. Field T, Hernandez Reif M, Diego M, Schanberg S, Kuhn C (2005) Cortisol decreases and serotonin and dopamine increase following massage therapy. The International Journal of Neuroscience 115(10): 1397-413.

24. Chung Y, Dumont RC (2011) Complementary and alternative therapies: Use in pediatric pulmonary medicine. Pediatric Pulmonary 46(6): 530544.

25. Cambron JA, Dexheimer J, Coe P, Swenson R (2007) Side-effects of massage therapy: A cross-sectional study of 100 clients. The Journal of Alternative and Complementary Medicine 13(8): 793-796.

26. Soligo M, Nori SL, Protto V, Florenzano F, Manni F (2013) Acupuncture and neurotrophin modulation. International Review of Neurobiology 111: $91-124$

27. Carroll R (2007) Endocrine System. Elsevier's Integrated Physiology. Elsevier Inc. Center for Disease Control (2018) CDC- Asthma-Data and Surveillance-Asthma Surveillance Data.

28. Lau K, Jones A (2008) A single session of Acu-TENS increases FEV and reduces dyspnoea in patients with chronic obstructive pulmonary disease: a randomized, placebo-controlled trial. Aust J Physiother 54(3): 179-184.

29. Brinkhaus B, Roll S, Jena S, Adam D, Binting S, et al. (2017) Acupuncture in patients with allergic asthma: a randomized pragmatic trial. Journal of Alterative \& Complementary Medicine 23(4): 268-277.

30. Marino D, Marrara K, Di Lorenzo V, Marcelo L Silva, Josie Resende Torres Silva, et al. (2010) Short-term effects of acupuncture on pulmonary function and heart rate in patients with chronic obstructive pulmonary disease. Journal of Chinese Medicine 10: 18-19.

31. Ngai S, Jones A, Hui Chan C, Ko FW, Hui DS (2013) An adjunct intervention for management of acute exacerbation of chronic obstructive pulmonary disease. J Altern Complement Med 19(2): 178-181.

32. Academy of Guided Imagery (2019) How Imagery Works.

33. Ackerman CJ, Turkoski B (2000) Using guided imagery to reduce pain and anxiety: Home Healthcare Nurse 18(8): 524-530.

34. Farver Vestergaard I, Jacobsen D, Zachariea R (2015) Efficacy of psychosocial interventions on psychological and physical health outcomes in chronic obstructive pulmonary disease: A systematic review and meta-analysis. Psychotherapy \& Psychosomatics 84(1): 3750 .

35. White JD (2014) Complementary and Alternative Medicine. Abeloffs Clinical Oncology, ( $5^{\text {th }}$ edn $)$. Elseiver Inc, USA.

36. Hoffart M, Keene E (1998) Body-Mind-Spirit: The benefits of visualization. American Journal of Nursing 98(12): 44-47.

37. Au Buchon B (1990) The effects of positive mental imagery on hope, coping, anxiety, dyspnea and pulmonary function in persons with chronic obstructive pulmonary disease: Tests of a nursing intervention and a theoretical model. University Microfilms International. Arbor, MI, USA.

38. Louie S (2004) The effects of guided imagery relaxation in people with COPD. Occup Ther Int 11(3): 145-159. 
39. Freeman LW, Welton D (2005) Effects of imagery, critical thinking, and asthma education on symptoms and mood state in adult asthma patients: A pilot study. The Journal of Alternative \& Complementary Medicine 11(1): 57-68.

40. Epstein GN, Halper JP, Barrett EA, Birdsall C, Mc Gee M, et al. (2004). A pilot study of mind-body changes in adults with asthma who practice mental imagery. Alternatives Therapies 10(4): 66-71.

41. Lecomte T, Spidel A (2016) Is mindfulness useful or dangerous for individuals with psychosis? Visions 12(2): 10-13.
42. Wood D, Patricolo GE (2013) Using guided imagery in a hospital setting. Alternative \& Complementary Therapies 19(6): 301-305.

43. Hyland ME, Halpin DM, Blake S, Seamark C, Pinnuck M, et al. (2016) Preference for different relaxation techniques by COPD patients: comparison between six techniques. International Journal of Chronic Obstructive Pulmonary Disease 11(1): 2315-2319.

44. Lillis C (2018) What is a TENS unit, and does it work? Medical News Today. 